\title{
Acquired hepatocerebral degeneration
}

\author{
Destînâ Yalçın, MD, Emel Ŏguz-Akarsu, MD, Mehmet Sökmen, MD.
}

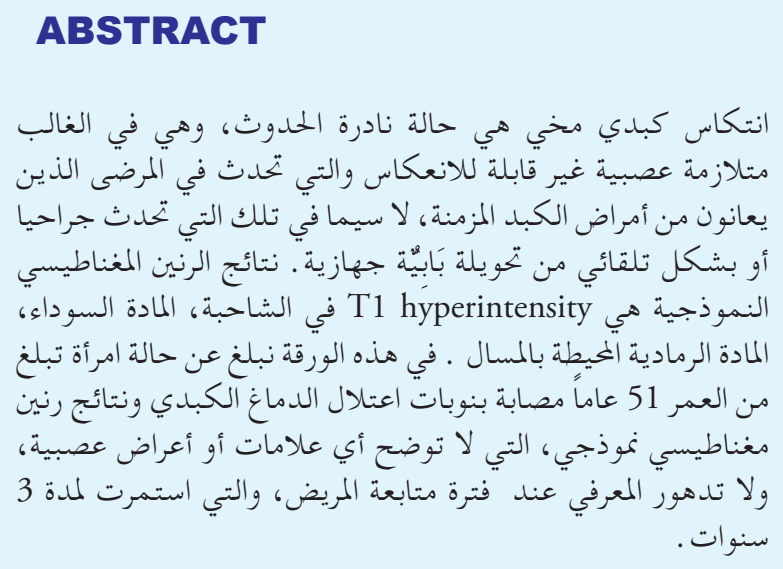

Acquired hepatocerebral degeneration is a rare, mostly irreversible neurological syndrome that occurs in patients with chronic liver disease, particularly in those with surgically or spontaneously induced portosystemic shunts. Typical magnetic resonance findings are T1 hyperintensity in the pallidum, substantia nigra, periaquaductal gray matter. In this paper, we report a case of a 51-year-old woman presented with hepatic encephalopathy episodes and typical magnetic resonance findings, who does not develop any neurological signs or symptoms, nor cognitive decline in the follow up period, lasting for 3 years.

Neurosciences 2016; Vol. 21 (2): 164-167 doi: 10.17712/nsj.2016.2.20150164

From the Neurology Clinic (Yalçın, Akarsu), and Gastroenterology Clinic (Sökmen), Umraniye Research and Training Hospital, Istanbul, Turkey.

Received 4th March 2015. Accepted 24th June 2015.

Address correspondence and reprint request to: Dr. Destînâ Yalçın, Associate Professor, Neurology Clinic, Ümraniye Research and Training Hospital, Istanbul, Turkey.E-mail: destinayalcin@yahoo.com

$\mathrm{A}^{\mathrm{c}}$ cquired hepatocerebral degeneration (AHD) is a rare, mostly irreversible neurological syndrome that occurs in patients with chronic liver disease, particularly in those with surgically or spontaneously induced portosystemic shunts. It was first described in 1914 by Van Woerkem, ${ }^{1}$ but remained largely unrecognized until the review by Victor et $\mathrm{al}^{2}$ in 1965 which has been a milestone in the history of the disease. ${ }^{3}$ The core features of AHD include extrapyramidal signs (Parkinsonism, tremor, chorea, athetosis, myoclonus, and astexisis), ataxia and cognitive decline. ${ }^{3}$ Additionally, patients may have neuropsychiatric changes, such as delirium, apathy, lethargy, somnolence, and emotional instability. ${ }^{4,5}$ Typical magnetic resonance (MR) findings are T1 hyper-intensity in the pallidum, substantia nigra, periaqueductal gray matter, and sometimes dentate nucleus. ${ }^{6}$ In this paper, we report a case of a 51 year-old woman presented with hepatic encephalopathy (HE) episodes and typical MR findings who does not develop any neurological signs or symptoms nor cognitive decline in the follow-up period, lasting for 3 years.

Case Report. The patient visited our emergency department after an episode which lasted for one day. It was characterized by increased drowsiness, staring, repeating the same movement over and over again, and asking the same questions several times. Her neurological examination was normal, but she was amnestic during the confusional state. She had experienced a similar episode 1 year ago and stated that she could not remember anything regarding the episode. When she was asked for any triggering factor for these attacks, she said that she had suffered from influenza with subfebrile fever in both incidences. Her medical record included Budd-Chiari syndrome since 17 years and she was operated on transjugular intrahepatic portosystemic shunt (TIPS) 5 years ago because -of recurrent esophageal variceal bleeding. Medical history of her family included one sister who suffered from juvenile myoclonic epilepsy. Her liver function tests (aspartate aminotransferase: $32 \mathrm{U} / \mathrm{L}$ normal: 5-34 U/L, alanine aminotransferase $33 \mathrm{U} / \mathrm{L}$ normal 0-55 U/L, gammaglutamyl transferase: $34 \mathrm{U} / \mathrm{L}$ normal: 9-36 U/L) were normal. Her hepatitis markers, including $\mathrm{HBsAg}$, anti-HBs, anti-HCV, and anti-HIV were negative. Moreover, prothrombin time was $22.2 \mathrm{sec}$ (normal:11$15 \mathrm{sec}$ ), activated partial thromboplastin time was 41.2 
sec (normal: 25-40 sec) because she was under warfarin treatment. Further laboratory investigations for Wilson's disease, which was considered in the differential diagnosis, revealed normal 24 hours urine copper level (48 microgram/24 hours normal: 3-50) and normal blood ceruloplasmin $(23 \mathrm{mg} / \mathrm{dL}$ normal: 18.9-148.7 $\mathrm{mg} / \mathrm{dL}$ ). No Kayser-Fleischer rings were observed in eye examination. Her Electroencephalography (EEG), recorded in a private hospital during the last attack showed diffuse slow waves in theta range intermixed with triphasic waves, suggesting primarily a hepatic encephalopathy (Figure 1). Her cranial MR revealed hyper-intense signals in globus pallidus, substantia nigra and dentate nuclei bilaterally, compatible with AHD (Figure $2 \& 3$ ). The EEG repeated one day after the episode, with negative neurological examination, showed slow waves in theta range $(7-7,5 \mathrm{cys} / \mathrm{sec})$ but no triphasic waves (Figure 4). She was followed for 3 years regularly with repeated neurological examination and EEGs, and remained healthy without any pathological signs or symptoms in her neurological examination. She is still active, working without any decline in her cognitive performance. Her repeated EEGs did not show any triphasic waves.

Discussion. The relationship between the brain and the liver has been well established since many years.
Among the neurological problems based on chronic liver diseases, the most common and widely recognized one is the reversible syndrome of $\mathrm{HE}$ which can be divided into 2 main forms: minimal $\mathrm{HE}$ and overt HE. ${ }^{3,7}$ The first one, which used to be referred to as latent or subclinical HE according to the old terminology, is now replaced with the term minimal HE. It is characterized by mild impairment of visuospatial function, attention and psychomotor speed. ${ }^{7,8}$ The second form is referred to as a syndrome of neuropsychiatric, neuropsychological and neurological disturbances arising as a complication of liver disease. Despite previous studies suggesting the condition is reversible, there is growing evidence that reversibility is incomplete. ${ }^{7,8}$

Both confusion episodes of our patient were compatible with overt HE attacks. However, it is noteworthy that in the follow-up period lasting for 3 years, neither of these episodes repeated nor the patient developed any neurological sign or symptom or impairment of cognitive functions. She even got a promotion which is the best evidence of her successful cognitive performance. The absence of liver cirrhosis could be the reason for the benign course of the disease, since most of the cases diagnosed AHD and described in the literature were suffering from cirrhosis but further investigation are necessary to confirm this hypothesis. ${ }^{8}$ The pathogenesis of the disease is poorly understood, but it is assumed that the chronic exposure of the brain

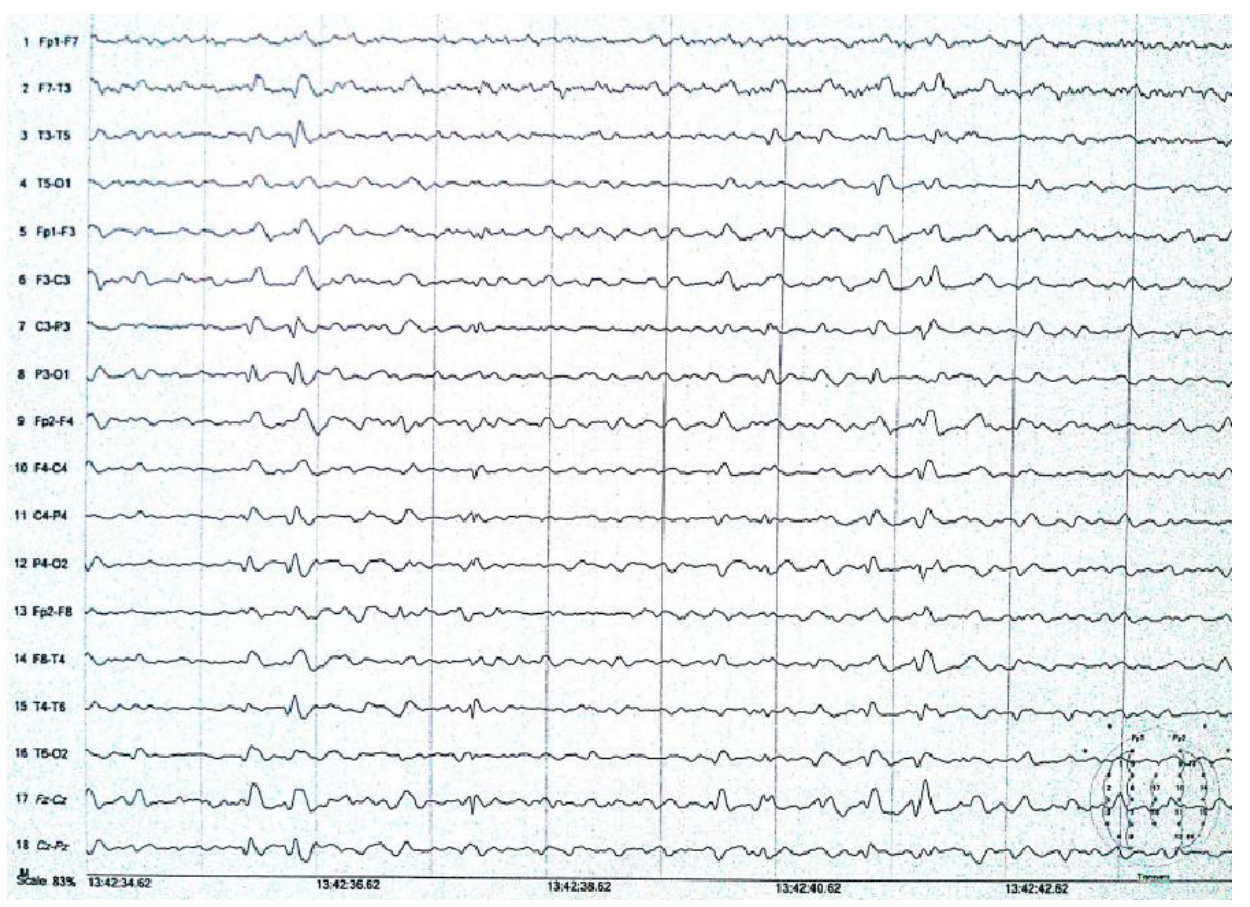

Figure 1 - The electroencephalography during the confusion attack showing triphasic waves. 
to toxic substances, especially to manganese, bypassing the liver is the main cause of the disease. ${ }^{3,9}$ Typical MR findings show an abnormally high signal on T1-weighted imaging in the basal ganglia, particularly globus pallidus. This high signal is now believed to be due to manganese deposition, and postmortem studies

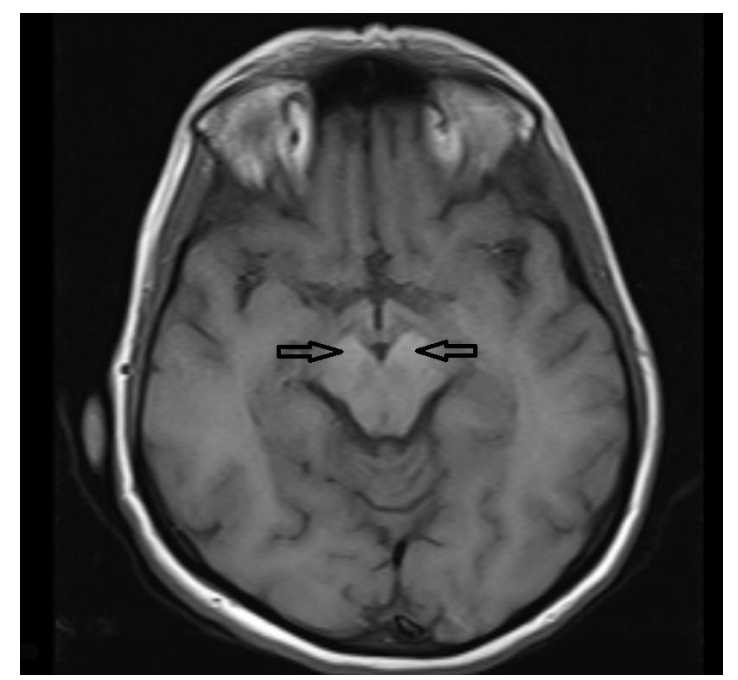

Figure 2 - T1-weighted axial magnetic resonance image shows bilateral symmetrical abnormal hyperintensity in the substantia nigra. have shown levels up to 7 times normal in globus pallidus confirming the main role of manganese in the pathogenesis of the disease. ${ }^{8}$ Magnetic resonance imaging findings of our patient were typical for AHD.

Although AHD has been long assumed to be irreversible, growing evidence shows that orthotropic

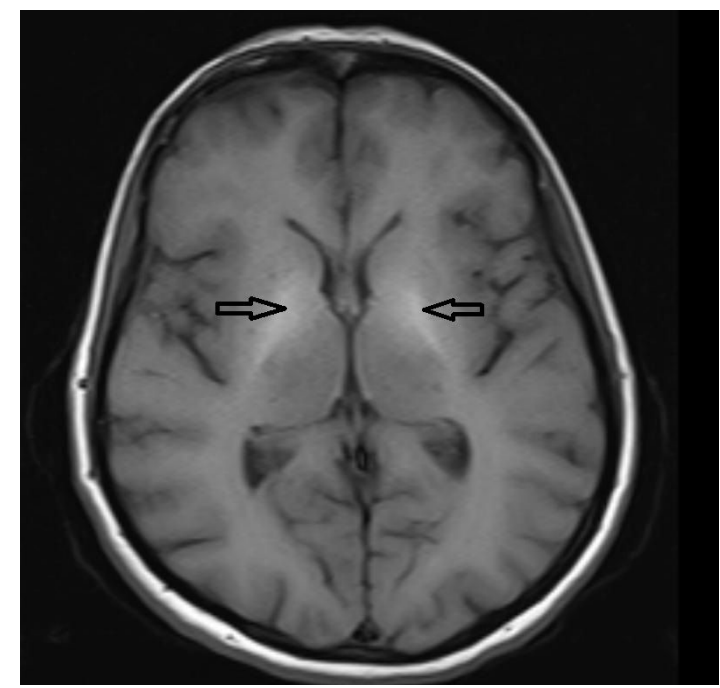

Figure 3 - T1-weighted axial magnetic resonance image shows bilateral symmetrical abnormal hyperintensity in the globus pallidus.

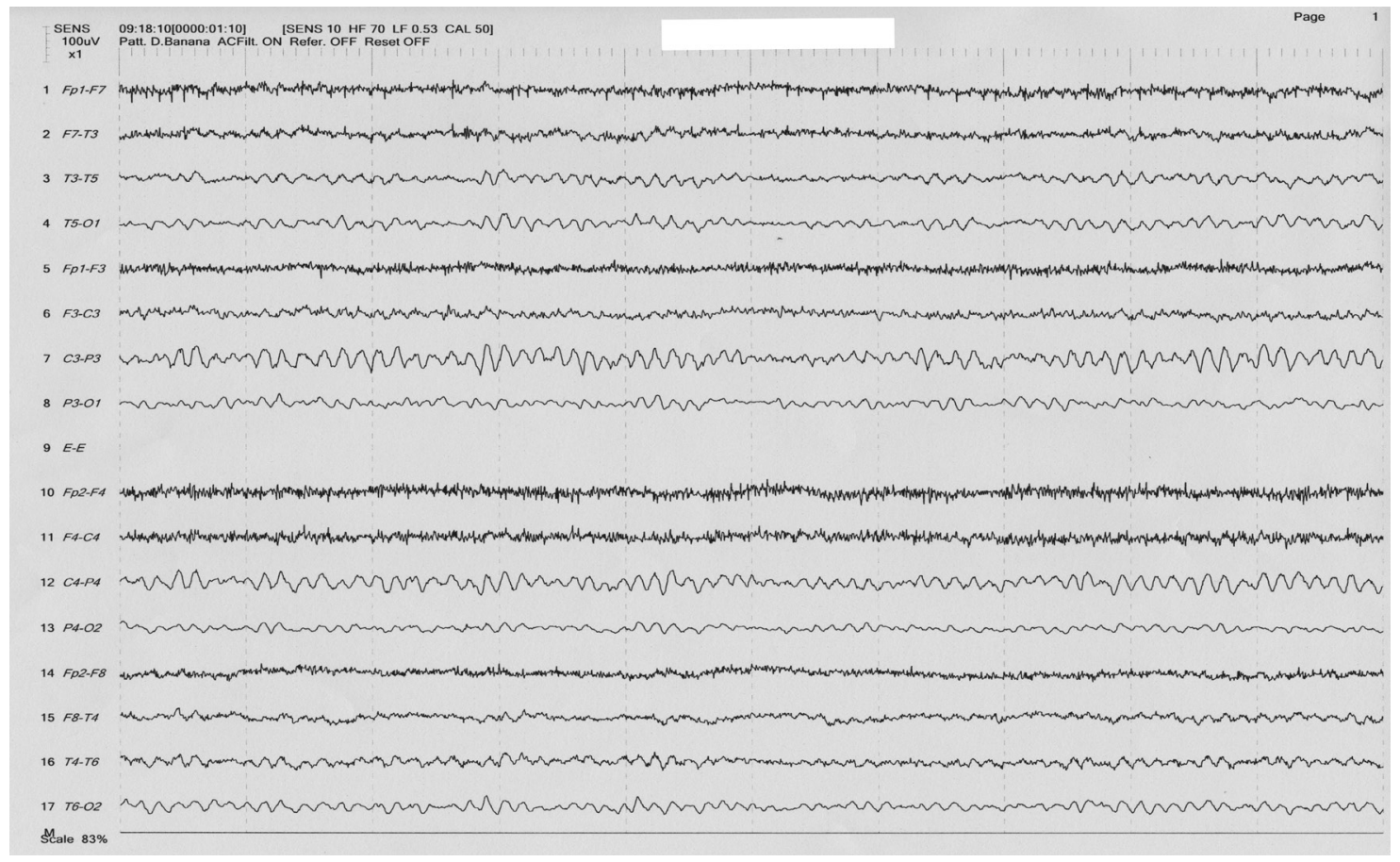

Figure 4 - The electroencephalography recorded one day after the episode showed slow waves in theta range (7-7,5 cys/ sec) but no triphasic waves. 
liver transplantation (OLT) is the best treatment option which improves both motor and cognitive functions. ${ }^{3,10}$ Additionally, patients who could not be submitted to OLT can be treated by reducing the flow through the portosystemic shunts or by obliteration of them. ${ }^{11}$ The MRI findings, medical history including Budd-Chiari syndrome and operation on TIPS, overt HE attacks with triphasic waves on EEG are all in accordance with AHD.

In conclusion, we would like to draw attention to the time course of the disease; the time between the outset of the degenerative process in the brain during the disease and the clinical manifestation of typical picture can take many years. Because of the lack of a gold standard for assessing AHD, we propose to make cranial MRI of patients that represent with confusion state, especially those with triphasic waves in the EEG and surgically or spontaneously induced portosystemic shunts in the past history, in order to detect candidates of AHD. Finally, we hope that early diagnosis of AHD and prospective follow-up of patients will add crucial information about the course of the disease and make it possible to develop effective treatment strategies.

\section{References}

1. Van Woerkem W. La cirrhose hepatique avec alterations dans les centres nerveux evoluant chez des sujets d'age moyen. Nouvelle Iconographie de la Salpetriere. Clinique des Maladies du Systeme Nerveux 1914; 7: 41-51.

2. Victor M, Adams RD, Cole M. The acquired (non-Wilsonian) type of chronic hepatocerebral degeneration. Medicine (Baltimore) 1965; 44: 345-396.
3 Ferrara J, Jankovic J. Acquired hepatocerebral degeneration. J Neurol 2009; 256: 320-332.

4. Gleason A, Hayhow B, Walterfang M, Evans E, Mocellin R, Gates P, et al. Neuropsychiatric symptoms as the presenting feature of acquired hepatocerebral degeneration. Aust $N Z J$ Psychiatry 2014; 48: 959-960.

5. Chen Y, Haque M, Yashida EM. Transient improvement of acquired hepatocerebral degeneration with parkinsonian symptoms after failed liver transplant: case report and literature review. Exp Clin Transplant 2011; 9: 363-369.

6. Maffeo E, Montuschi A, Stura G, Giordana MT. Chronic acquired hepatocerebral degeneration, pallidal T1 MRI hyperintensity and manganese in a series of cirrhotic patients. Neuro Sci 2014, 35: 523-530.

7. Lewis M, Howdle PD. The neurology of liver failure. QJM 2003; 96: 623-633.

8. Romeiro FG, Américo MF, Yamashiro FS, Caramori CA, Schelp AO, Antonio C, et al. Acquired hepatocerebral degeneration and hepatic encephalopathy. Arq Neuropsiquiatr 2011; 69: 496-501.

9. Baheti NN, Hassan H, Rathore C, Krishnan S, Kesavadas C. Acquired hepatolenticular degeneration: is the T1 hyperintensity due to manganese deposition? Neurol India 2009; 57: 812-813.

10. Stracciari A, Baldin E, Cretella L, Delaj L, D'Alessandro R, Guarino M. Chronic acquired hepatocerebral degeneration: effects of liver transplantation on neurological manifestations. Neurol Sci 2011; 32: 411-415.

11. Hisahara S, Matsushita T, Kitamura M, Mezawa S, Nonaka $\mathrm{M}$, Imai $\mathrm{T}$, et al. long-term clinical and radiological improvement of chronic acquired hepatocerebral degeneration after obliteration of portosystemic shunt: report of a case. J Neurol Sci 2014; 346: 303-306.

\section{NEW PEER REVIEWERS}

Join our team of expert peer reviewers for the Neurosciences Journal by sending an enquiry and summarized CV to info@smj.org.sa. Note that NSJ reviewers, whose reviews are returned on time and are judged satisfactory by the Editors, may receive $1 \mathrm{CME}$ credit per review, with a maximum of 5 credits per year, from the Saudi Council for Health Specialties. 